\title{
On the Flexibility of Metal-Organic Frameworks
}

\author{
Lev Sarkisov, ${ }^{* \dagger}{ }^{\dagger}$ Richard L. Martin, ${ }^{\ddagger}$ Maciej Haranczyk, ${ }^{\ddagger}$ and Berend Smit* ${ }^{*}, \|$ \\ ${ }^{\dagger}$ Institute for Materials and Processes, School of Engineering, The University of Edinburgh, Edinburgh EH9 3JL, United Kingdom \\ ${ }^{\ddagger}$ Computational Research Division and "Materials Sciences Division, Lawrence Berkeley National Laboratory, Berkeley, California \\ 94720-8139, United States \\ ${ }^{\S}$ Department of Chemical and Biomolecular Engineering, University of California, Berkeley, California 94720, United States
}

\section{Supporting Information}

ABSTRACT: Occasional, large amplitude flexibility in metal-organic frameworks (MOFs) is one of the most intriguing recent discoveries in chemistry and material science. Yet, there is at present no theoretical framework that permits the identification of flexible structures in the rapidly expanding universe of MOFs. Here, we propose a simple method to predict whether a MOF is flexible, based on treating it as a system of rigid elements, connected by hinges. This proposition is correct in application to MOFs based on rigid carboxylate linkers. We validate the method by correctly classifying known experimental MOFs into rigid and flexible groups. Applied to hypothetical MOFs, the method reveals an abundance of flexibility phenomena, and this seems to be at odds with the proportion of flexible structures among experimentally known MOFs. We speculate that the flexibility of a MOF may constitute an intrinsic impediment on its experimental realization. This highlights the importance of systematic prediction of large amplitude flexibility regimes in MOFs.

$\mathrm{F}$ lexibility of metal-organic frameworks (MOFs) has - evolved from a curiosity to an integral part of MOF chemistry. ${ }^{1,2}$ Indeed, over the years an increasing number of MOFs have been discovered which exhibit a diverse range of conformational effects, from negative thermal expansion to large amplitude reversible deformations, such as breathing effects and swelling. ${ }^{3-6}$ The interest in flexibility is motivated by its potential exploitation in developing stimuli-responsive MOFs for controlled drug release and sensing applications. ${ }^{7,8}$ In other applications, such as industrial adsorption separation processes, excessive deformation of adsorbents may not be desirable, as this would lead to attrition and eventual disintegration of the adsorbent. These applications rely on a fundamental understanding of the molecular factors that determine the flexibility of MOFs. The current practice to discover flexibility of novel structures is through experiments. Depending on the material, chemical (adsorption), thermal, or mechanical external stimuli are applied to induce deformations, with several complementary techniques employed to understand the elastic response of a MOF. ${ }^{6,9-15}$ Molecular simulations using atomistic force fields or $a b$ initio quantum mechanical calculations have been extremely helpful in explaining flexibility effects, ${ }^{6,15-21}$ but these methods typically require prior knowledge of modes of flexibility from experi- ments. Given the large number of experimentally known MOFs and virtually infinite number of hypothetical structures, current theoretical methods seem impracticable for high-throughput predictive analysis. Is it possible to predict or anticipate large flexibility effects in a MOF simply from its crystal topology?

In this work we develop a simple and computationally efficient method to address this challenge. The central proposition is that the structure of MOFs can be considered as a mechanical network of rigid bodies, connected by flexible joints, and that large scale flexibility in MOFs is governed by the cooperative effects in this network. Below we demonstrate the accuracy of this proposition in application to the most common class of MOFs based on carboxylate linkers; however the proposed approach is very general and can be easily extended to other classes of MOFs.

Indeed, many MOFs are metal-oxygen clusters connected via organic linkers. Clusters and linkers can be viewed as relatively rigid elements. However, at the point where these elements are connected to each other, they retain some degree of freedom. In the terminology of Férey and Serre, these connections are called "weak points", and for the MOF crystal to be flexible, certain symmetry in their location must be obeyed. ${ }^{2}$ In order to construct mechanical models of MOFs, the properties of the connection between rigid elements must be correctly represented.

Consider a single crystal cage of IRMOF-1 (Figure 1), consisting of octahedral secondary building units (SBUs) of four $\mathrm{ZnO}_{4}$ tetrahedra, connected by benzene dicarboxylate (BDC) linkers. In the reduced topological representation of Yaghi et al., ${ }^{22,23}$ IRMOF-1 consists of vertices with six extension points linked by bars (Figure 1). This approach makes it possible to classify MOFs by their topology, but it does not distinguish the nature of the connections.

Investigating these connections in more detail, two oxygen atoms and the carbon atom of the linker form a rigid triangular structure, which can rotate (within certain limits) around the axis linking the oxygen atoms (Figures 1 and S1). In mechanical engineering terms this connection is called a hinge, and it constraints the movement of the linker to a plane. Therefore, flexibility (or lack thereof) of a MOF can be assessed by analyzing its structure as a system of rigid elements connected by hinges.

Received: November 15, 2013

Published: January 24, 2014 


\begin{tabular}{l|l|l|l|}
\hline Chemical & Topological & Mechanical \\
\hline
\end{tabular}

Figure 1. Different representations of IRMOF-1. Chemical representation is shown on the left, including a visualization of a single hinge connection between a metal cluster and carboxylate linker; reduced topological representation is shown in the center, following Yaghi et al.;2,23 and two mechanical representations are shown on the right, using the actual mechanical prototypes and systems of molecular trusses (far right). Color scheme for the chemical representation: cyan for carbon, gray for zinc, red for oxygen, and white for hydrogen.

From the mechanical point of view the simplest rigid element is a triangle, while in civil engineering, this element is called a truss, and the structural stability of an object, such as a bridge, can be reduced to the analysis of a system of trusses. Taking inspiration from both mechanical and civil engineering, we developed an algorithm to reduce MOFs to a system of molecular trusses. Two rigid elements, built from trusses, which share a common edge form a hinge connection and can rotate with respect to one another (Figure S2). We use the term "truss network" to describe a complete structure of a MOF. To visualize this concept, we can also construct an equivalent network by replacing the SBUs and linkers with rigid bodies connected by hinges. This "mechanical toy" representation is extremely helpful in explaining the principles of the approach here, as one can apply the deformation by hand.

For example, Figure 1 shows a single cage of IRMOF- 1 in the mechanical representation. The red cubes represent the SBUs and the blue rods the carboxylic linkers. The linkers connect to the red cubes via hinges oriented at $45^{\circ}$ with respect to the face of the IRMOF-1 cage. A single face of the IRMOF-1 cage, comprising four SBUs and four linkers, is very flexible in $3 \mathrm{D}$ (see Supporting Information, SI). Assembled together, however, the faces form a rigid three-dimensional network (Figure 1). Since each hinge restricts movement of the linkers to a plane, with all the hinges oriented at an angle to the faces of the cage they form a set of mutually exclusive degrees of freedom, which makes the structure rigid as a whole.

In the IRMOF-1-16 series of materials the topology of the structure and the metal cluster remain the same and only the linker changes. ${ }^{24}$ In our mechanical representation all these materials map onto the same truss network, hence establishing the rigidity of this whole class of MOFs.

In another example, the structure of MIL-53 can be considered as a system of metal-organic chains, connected by BDC linkers. As shown in Figure S7, in the mechanical representation, metal-oxygen chains become red rods and linkers become blue rods, attached to red rods by hinges. Within this structure the hinges are oriented parallel to one another. This allows concerted movement of all linkers in the same plane, resulting in high flexibility and the large breathing transitions observed in MIL-53. ${ }^{5}$

In the high-throughput flexibility analysis implemented here, the crystal structure of a MOF is, first, reduced to a system of molecular trusses, preserving periodic boundary conditions. Details of this procedure and examples of such mappings are provided in the SI file. Second, we analyze available conformations of the truss network, compatible with physically meaningful deformations. For this, bonds of the molecular trusses are described as stiff harmonic springs. The unit cell of the truss network is perturbed according to one of several available regimes, such as linear compression, shear deforma-

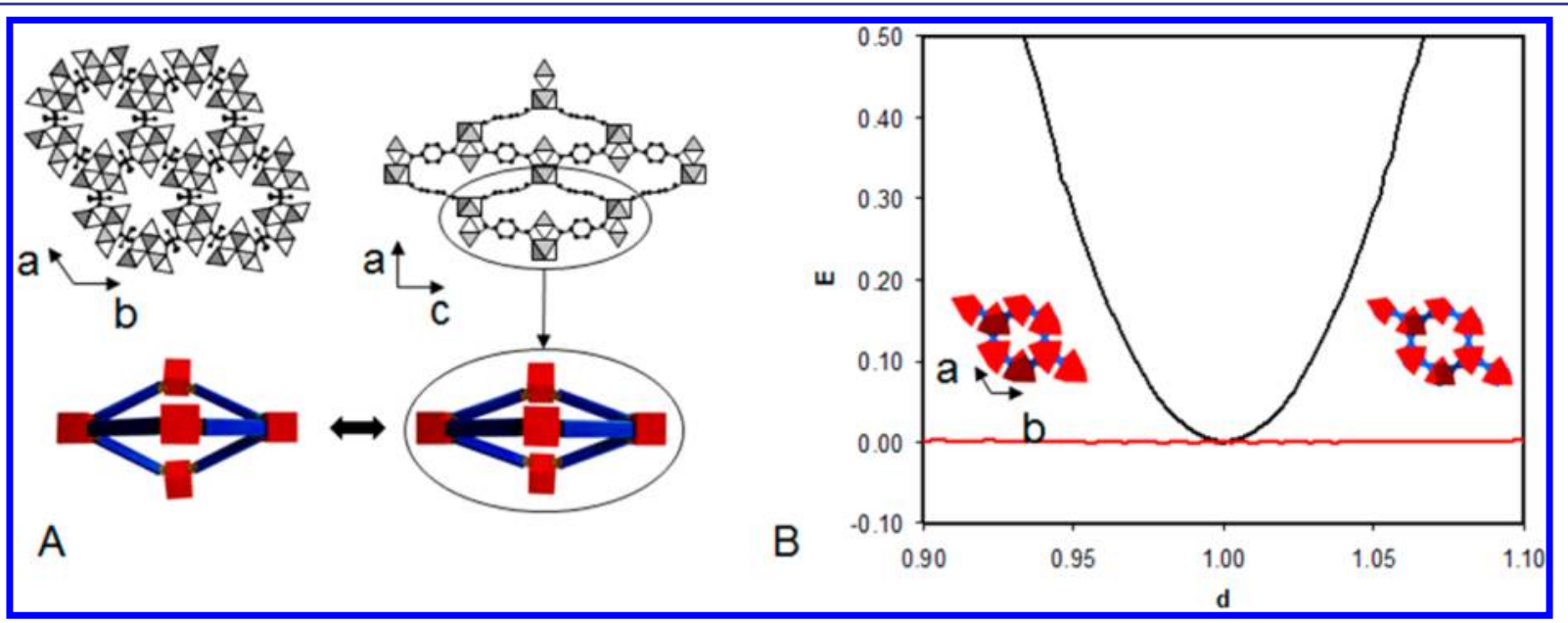

Figure 2. Flexibility analysis of the MIL- 88 family of materials. (A) Structure of MIL-88B from two different perspectives (top). It consists of trimers of metallic octahedra, joined by carboxylate organic linkers (in MIL-88B, BDC). Along the crystallographic $b$ axis (top, right), the structure can be seen to comprise bipyramids formed by five trimers of metallic octahedra and six BDC linkers. On the bottom the figure shows a mechanical representation of a single bipyramid which is able to flex as shown along the axis connecting tips of the pyramids. (B) Energy penalty $E$ as a function of order parameter $d$ for selected perturbations. Black line corresponds to perturbation of $\beta$ unit cell angle; red line corresponds to compressionexpansion of $a$ and $b$ lattice vectors with simultaneous relaxation in $c$. Snapshots on the left and right illustrate the geometry of the system, corresponding to the extreme points on the red line $(d=0.90$ and 1.10$)$. 
tion, or a combination thereof. This perturbation is described by the order parameter, $d$, which characterizes the deviation of the lattice parameter(s) of the unit cell from the original value(s). Steepest descent energy minimization is used to find the conformation of the network within the deformed unit cell. A flexible network, compatible with a particular deformation mode, should exhibit a very small energy penalty. By contrast, a rigid network will only be able to adopt the new shape through substantial expansion (contraction) of the links, associated with a large energy penalty.

This approach constitutes a computationally efficient and force field-independent framework, within which flexibility of various classes of MOFs can be enumerated. To demonstrate this, in the SI file we return to the example of MIL-53 (Figure S11). We show typical responses of the energy penalty when the system behaves as a rigid structure in response to shear by changing the $\alpha$ angle of the unit cell, and when it is able to flex under compression-expansion of the $b$ lattice vector with a simultaneous relaxation of the $c$ lattice vector.

The complete set of 13 deformation regimes explored includes a separate perturbation of each lattice parameter of the unit cell and combinations of perturbations, summarized in the SI file. Within this approach, IRMOF-1, HKUST-1 (subtle negative thermal expansion effects in HKUST-1 are beyond the scope or resolution of the simplified approach here), MOF-180, MIL-74, MOF-36, and NU-110 have been correctly identified as rigid whereas MIL-47 and MIL-53 showed the expected large amplitude flexible modes (see Table S6). It is important to note here that our approach is meant to detect potential flexibility only, while molecular details and energy landscape of the deformations should be properly investigated in the follow-up quantum mechanical studies. For example, although MIL-47, MIL-53, and Co(1,4-benzenedipyrazolate) are topologically similar, their experimentally observed behavior is quite different. MIL-47 does not exhibit breathing behavior upon adsorption, whereas MIL-53 does and Co(1,4-benzenedipyrazolate) is uniquely capable of a five-step breathing transition upon nitrogen adsorption. ${ }^{25}$ These differences are beyond the scope of the approach here.

MIL-53 nicely illustrates the concept, but this example is also somewhat deceptive, as in this case the topology is sufficiently simple to anticipate the cooperative effects directly from its visual inspection (one may think of so-called "wine rack" model in the terminology of Coudert et al.). ${ }^{26}$ For other materials this is not so straightforward. For example, a combination of powder diffraction experiments and modeling was required to explain large-scale swelling in the MIL-88 family of materials. ${ }^{6,27}$ As seen from the schematic depiction in Figure 2A, MIL-88B consists of trimers of metallic octahedra joined by carboxylate organic linkers. All structures within the MIL-88 series share the same pore topology. Along the $c(z)$ axis of the unit cell, MIL-88B features a hexagonal arrangement of narrow pores. Viewed along the crystallographic $b$ axis (Figure 2A, right), the structure can be seen as a system of triangular bipyramids, whose vertices are trimers of the metallic octahedra and whose edges are carboxylate linkers. Taking our mechanical point of view, the observed flexibility can be explained using a model of a single bipyramid, where vertices and linkers are represented as rigid bodies connected by hinges (Figure 2A). This mechanical model can be easily squeezed or stretched along the axis connecting the two extreme tips of the bipyramid. During this process the other three vertices forming the face shared by the pyramids remain in the same plane but are either drawn further apart or brought closer together, respectively.

Deformation analysis of a complete periodic system of molecular trusses of MIL-88 topology captures this behavior automatically. From Figure 2B, MIL- 88 shows clear flexibility under isotropic compression-expansion of $a$ and $b$ lattice vectors with simultaneous relaxation of the system in $c$. In addition, Figure S14 shows changes in the lattice vector $c$ and volume of the unit cell (normalized with the values of these parameters in the initial crystallographic structure) as a function of normalized lattice vector $a$. Indeed, as the system is squeezed along the $a$ and $b$ vectors it slightly expands in $c$. In the same graph we also plot the experimental data for MIL-88A, from the original study of Mellot-Draznieks et al. ${ }^{6}$ The agreement between the data illustrates that the swelling behavior of the crystal cell can be predicted with high accuracy based on purely mechanical grounds, as was previously noted by Férey and Serre. $^{2}$

Experimental studies on MOFs with tailored mechanics have already started to emerge. ${ }^{28,29}$ Our algorithm can be used to guide the design of MOFs with specific rigidity properties. To illustrate its practical importance, it is instructive to revisit the computational enumeration and screening of MOFs for methane storage as pioneered by Wilmer et al. ${ }^{30}$ Again, for methane storage large amplitude flexibility may not be desirable, as this would also lead to material attrition and substantially complicate the design of storage tanks. Our analysis extended to the proposed top 50 MOFs with the highest volumetric storage suggests, surprisingly, that only one is rigid (see SI).

This result poses the intriguing question of how common flexibility is in MOFs. Is the substantial presence of flexible structures in the database of Wilmer et al. a result of a rather small number of underlying nets as has been recently discussed $?^{31}$ To answer this question, we utilized experimentally realized metal clusters and carboxylate linkers of the required coordination to assemble hypothetical MOFs within 13 high-symmetry topologies, as suggested by DelgadoFriedrichs et al. ${ }^{32,33}$ Our analysis (Table 1, details in SI) showed that out of 13 hypothetical MOFs, only 4 were properly rigid, whereas 7 were flexible and 2 showed signs of limited flexibility. Visualization of the flexible modes suggests that they can be surprisingly complex, and it would be nearly

Table 1. Summary of Investigated Hypothetical MOFs ${ }^{a}$

$\begin{array}{cccc}\text { net } & \mathrm{SBU} & \text { linker } & \text { flexible } \\ \text { acs } & \mathrm{Pd}_{3} & \mathrm{BDC} & \text { yes } \\ \text { bor } & \mathrm{V}_{4}(\mathrm{OH})_{4} & \mathrm{BTC} & \text { no } \\ \text { cds } & \mathrm{Cu}_{2} & \mathrm{BDC} & \text { yes } \\ \text { dia } & \mathrm{V}_{4}(\mathrm{OH})_{4} & \mathrm{BDC} & \text { yes } \\ \text { hxg } & \mathrm{Ti}_{6} \mathrm{O}_{6} & \mathrm{BDC} & \text { no } \\ \text { lvt } & \mathrm{Cu}_{2} & \mathrm{BDC} & \text { yes } \\ \text { nbo } & \mathrm{Cu}_{2} & \mathrm{BDC} & \text { yes } \\ \text { pcu } & \mathrm{Zn}_{4} \mathrm{O} & \mathrm{BDC} & \text { semi } \\ \text { pto } & \mathrm{Cu}_{2} & \mathrm{BTC} & \text { semi } \\ \text { pyr } & \mathrm{Zn}_{4} \mathrm{O} & \mathrm{BTC} & \text { no } \\ \text { rhr } & \mathrm{Cu}_{2} & \mathrm{BDC} & \text { yes } \\ \text { she } & \mathrm{Cu}_{2} & \mathrm{BHC} & \text { no } \\ \text { sod } & \mathrm{V}_{4}(\mathrm{OH})_{4} & \mathrm{BDC} & \text { yes }\end{array}$

${ }^{a}$ All nets described are available from the Reticular Chemistry Structural Resource. ${ }^{32,33}$ 
impossible to anticipate them simply from visual inspection of the crystal structure (for a complete analysis and visualization of all flexible regimes for all structures considered here see SI and http://www.nanoporousmaterials.org/flexibility).

This leads to two important concepts. On one hand, the proposed algorithm can be used to construct databases of hypothetical MOFs, with an additional imposed constraint for the MOFs to be rigid. For example, the four hypothetical MOFs identified as rigid suggest general truss networks which can be used as templates to construct other rigid MOFs by varying the linkers. On the other hand, we do not observe as many very flexible MOFs among experimental structures compared to hypothetical structures from our analysis or that of Wilmer et al. Aside from high symmetry of our selection of nets, a tempting explanation is that flexible MOFs are intrinsically much more difficult to synthesize due to structural instability. With insights about large amplitude flexibility we obtained and the resulting algorithms, crystal engineering of flexible and rigid MOFs can now be guided in a systematic way.

\section{ASSOCIATED CONTENT}

\section{S Supporting Information}

Mechanical models of MOFs, computational algorithm details, results for all the structures explored, computer visualizations, reference to the associated web resources. This material is available free of charge via the Internet at http://pubs.acs.org.

\section{AUTHOR INFORMATION}

\section{Corresponding Authors}

Lev.Sarkisov@ed.ac.uk

Berend-Smit@berkeley.edu

Notes

The authors declare no competing financial interest.

\section{ACKNOWLEDGMENTS}

L.S. acknowledges financial support from the Royal Academy of Engineering and the Leverhulme Trust through the Senior Research Fellowship program. We thank Prof. Ileana Streinu for useful insights on the rigidity theory. R.L.M., M.H., and B.S. were supported by the U.S. Department of Energy, Office of Basic Energy Sciences, Division of Chemical Sciences, Geosciences and Biosciences under Award DE-FG02-12ER16362.

\section{REFERENCES}

(1) Kitagawa, S.; Uemura, K.. Chem. Soc. Rev. 2005, 34, 109.

(2) Férey, G.; Serre, C. Chem. Soc. Rev. 2009, 38, 1380.

(3) Wu, Y.; Kobayashi, A.; Halder, G. J.; Peterson, V. K.; Chapman, K. W.; Lock, N.; Southon, P. D.; Kepert, C. J. Angew. Chem., Int. Ed. 2008, 47, 8929.

(4) Barthelet, K.; Marrot, J.; Riou, D.; Férey, G. Angew. Chem., Int. Ed. 2001, 41, 281.

(5) Serre, C.; Millange, F.; Thouvenot, C.; Nogues, M.; Marsolier, G.; Louer, D.; Férey, G. I. Am. Chem. Soc. 2002, 124, 13519.

(6) Mellot-Draznieks, C.; Serre, C.; Surble, S.; Audebrand, N.; Férey, G. I. Am. Chem. Soc. 2005, 127, 16273.

(7) Keskin, S.; Kizilel, S. Ind. Eng. Chem. Res. 2011, 50, 1799.

(8) Horcajada, P.; Gref, R.; Baati, T.; Allan, P. K.; Maurin, G.; Couvreur, P.; Férey, G.; Morris, R. E.; Serre, C. Chem. Rev. 2012, 112, 1232.

(9) Loiseau, T.; Serre, C.; Huguenard, C.; Fink, G.; Taulelle, F.; Henry, M.; Bataille, T.; Férey, G. Chem.-Eur. I. 2004, 10, 1373.

(10) Boutin, A.; Springuel-Huet, M. A.; Nossov, A.; Gedeon, A.; Loiseau, T.; Volkringer, C.; Férey, G.; Coudert, F. X.; Fuchs, A. H. Angew. Chem. Int. Ed. 2009, 48, 8314.
(11) Finsy, V.; Kirschhock, C. E. A.; Vedts, G.; Maes, M.; Alaerts, L.; De Vos, D. E.; Baron, G. V.; Denayer, J. F. M. Chem.—Eur. J. 2009, 15, 7724.

(12) Boutin, A.; Coudert, F. X.; Springuel-Huet, M. A.; Neimark, A. V.; Férey, G.; Fuchs, A. H. I. Phvs. Chem. C 2010, 114, 22237.

(13) Mowat, J. P. S.; Seymour, V. R.; Griffin, J. M.; Thompson, S. P.; Slawin, A. M. Z.; Fairen-Jimenez, D.; Düren, T.; Ashbrook, S. E.; Wright, P. A. Dalton Trans. 2012, 41, 3937.

(14) Yot, P. G.; Ma, Q. T.; Haines, J.; Yang, Q. Y.; Ghoufi, A.; Devic, T.; Serre, C.; Dmitriev, V.; Férey, G.; Zhong, C. L.; Maurin, G. Chem. Sci. 2012, 3, 1100.

(15) Llewellyn, P. L.; Maurin, G.; Devic, T.; Loera-Serna, S.; Rosenbach, N.; Serre, C.; Bourrelly, S.; Horcajada, P.; Filinchuk, Y.; Férey, G. I. Am. Chem. Soc. 2008, 130, 12808.

(16) Salles, F.; Ghoufi, A.; Maurin, G.; Bell, R. G.; Mellot-Draznieks, C.; Férey, G. Angew. Chem.. Int. Ed. 2008, 47, 8487.

(17) Ghoufi, A.; Maurin, G. I. Phvs. Chem. C 2010, 114, 6496.

(18) Fairen-Jimenez, D.; Moggach, S. A.; Wharmby, M. T.; Wright, P. A.; Parsons, S.; Düren, T. I. Am. Chem. Soc. 2011, 133, 8900.

(19) Triguero, C.; Coudert, F. X.; Boutin, A.; Fuchs, A. H.; Neimark, A. V. I. Phvs. Chem. Lett. 2011, 2, 2033.

(20) Ortiz, A. U.; Boutin, A.; Fuchs, A. H.; Coudert, F. X. Phys. Rev. Lett. 2012, 109, 195502-1.

(21) Chen, L.; Mowat, J. P. S.; Fairen-Jimenez, D.; Morrison, C. A.; Thompson, S. P.; Wright, P. A.; Düren, T. J. Am. Chem. Soc. 2013, 135, 15763.

(22) Ockwig, N. W.; Delgado-Friedrichs, O.; O’Keeffe, M.; Yaghi, O. M. Acc. Chem. Res. 2005, 38, 176.

(23) O'Keeffe, M.; Yaghi, O. M. Chem. Rev. 2012, 112, 675.

(24) Eddaoudi, M.; Kim, J.; Rosi, N.; Vodak, D.; Wachter, J.; O’Keeffe, M.; Yaghi, O. M. Science 2002, 295, 469.

(25) Salles, F.; Maurin, G.; Serre, C.; Llewellyn, P. L.; Knofel, C.; Choi, H. J.; Filinchuk, Y.; Oliviero, L.; Vimont, A.; Long, J. R.; Férey, G. I. Am. Chem. Soc. 2010, 132, 13782.

(26) Ortiz, A. U.; Boutin, A.; Fuchs, A. H.; Coudert, F.-X. J. Chem. Phys. 2013, 138, 174703.

(27) Surble, S.; Serre, C.; Mellot-Draznieks, C.; Millange, F.; Férey, G. Chem. Commun. 2006, 3, 284.

(28) Deng, H.; Olson, M. A.; Stoddart, J. F.; Yaghi, O. M. Nat. Chem. 2010, 2, 439.

(29) Ogborn, J. M.; Collings, I. E.; Moggach, S. A.; Thompson, A. L.; Goodwin, A. L. Chem. Sci. 2012, 3, 3011.

(30) Wilmer, C. E.; Leaf, M.; Lee, C. Y.; Farha, O. K.; Hauser, B. G.; Hupp, J. T.; Snurr, R. Q. Nat Chem. 2011, 4, 83.

(31) Sikora, B. J.; Winnegar, R.; Proserpio, D. M.; Snurr, R. Q. Microporous Mesoporous Mater. 2014, 86, 207.

(32) Delgado-Friedrichs, O.; O'Keeffe, M.; Yaghi, O. M. Phys. Chem. Chem. Phys. 2007, 9, 1035.

(33) O’Keeffe, M.; Peskov, M. A.; Ramsden, S. J.; Yaghi, O. M. Acc. Chem. Res. 2008, 41, 1782. 